Sylvia Gonçalves 1

Paulo Fagundes 1

Giovanni Lovisi 2

Lúcia Abelha Lima 2

\section{Avaliação das limitações no comportamento social em pacientes psiquiátricos de longa permanência ${ }^{\star}$}

The assessment of social role functioning impairments in long-stay psychiatric patients
* O presente estudo faz parte de uma pesquisa em andamento no Instituto

Municipal de Assistência à Saúde Juliano Moreira (IMASJM), da Secretaria Municipal de Saúde do Rio de Janeiro e no Hospital São Pedro Cidadão, da Secretaria Estadual de Saúde do Rio Grande do Sul, sob a chancela do Ministério da Saúde e da Organização Pan-Americana da Saúde e consultoria do professor Manoel Desviat e equipe de investigação do Instituto José Germain da Comunidade de Madri.

1 Núcleo de Documentação e Pesquisa/Centro de Estudos/Instituto Municipal de Assistência à Saúde Juliano Moreira. Estrada Rodrigues Caldas, 3.400 . 22713-370 - Jacarepaguá Rio de Janeiro.

sgoncalves@olimpo.com.br

2 Escola Nacional

de Saúde Pública,

Fundação Oswaldo Cruz.
Abstract This paper describes an assessment of social role functioning impairments in longstay psychiatric patients. Most recent studies have concentrated on this theme and these measures are very important to the transfer of care from hospitals to community facilities. In order to assess the long term impairments or disabilities, we have used the Social Behavioural Schedule (SBS) that covers behaviour areas which have been shown in previous research to describe the major difficulties exhibited by patients with long-term impairments. Forty-six women were randomized from a range of two hundred women living at the Nucleo Franco da Rocha, a hospitalar unit of Instituto Municipal Juliano Moreira in Rio de Janeiro. The NFR results were compared with a homeless hostels sample and a long stay wards sample, both in London. Despite the average age and the long term living in a psychiatric hospital, 1/3 of the studied population could live in supervised residences. These finds suggest that a significant percent of the patients who live in long stay psychiatric hospitals are able to live in community facilities.

Key words Social Behavioural Schedule, SBS, Deinstitutionalization, Social disability
Resumo Este texto trata de uma avaliação do comportamento social de pacientes psiquiátricos de longa permanência. Estudos recentes concentram-se nesse tema, considerando-o relevante na transferência do eixo da atenção do hospital para a comunidade. Para avaliar dificuldades e limitações de uma população com longo período de institucionalização, utiliza-se a Social Behavioural Schedule (SBS), que cobre áreas do comportamento referentes às principais dificuldades de pacientes crônicos. Selecionou-se uma amostra de 46 pacientes de uma população de 200 mulheres, com longa história de internação psiquiátrica, residentes em duas moradias protegidas e quatro pavilhões do Núcleo Franco da Rocha (NFR), do Instituto Municipal Juliano Moreira, no Rio de Janeiro. Os resultados obtidos foram comparados aos dados de uma amostra de moradores em um albergue de sem tetos e aos de pacientes de um hospital psiquiátrico, ambos situados em Londres. Os resultados sugerem que significativo percentual dos pacientes que vivem longo tempo em hospitais teriam condições de viver em residências na comunidade.

Palavras-chave Social Behavioural Schedule, SBS, Desinstitucionalização, Comportamento social 


\section{Introdução}

A partir da década de 1950 iniciou-se nos países desenvolvidos o processo de desinstitucionalização da assistência psiquiátrica, um movimento em prol da diminuição das internações psiquiátricas e do tratamento dos pacientes na comunidade. As principais razões que propiciaram seu surgimento foram as péssimas condições em que se encontravam os hospitais psiquiátricos, o movimento pelos direitos civis e pelos direitos humanos, o surgimento de psicofármacos mais eficazes - os neurolépticos - e o argumento de que o atendimento à saúde mental na comunidade teria um custo mais baixo (Bandeira,1991; Bassuk 1978; Fagundes, 1998; Hafner \& Heiden, 1989; Morgado \& Lima, 1994).

Via de regra, essas experiências não foram devidamente avaliadas no seu início, o que dificultou a análise dos processos e resultados da implantação dos novos serviços (Braun et al., 1981) e colocou em questão a necessidade de utilizar instrumentos capazes de medir o processo em todos os momentos para posteriormente possibilitar comparações que, consequentemente, permitiriam aferir a eficácia das intervenções de forma objetiva e confiável. Embora seja hegemônico o pensamento de que é melhor para os pacientes, do ponto de vista de qualidade de vida, serem tratados na comunidade, permanecem em aberto diversas questões relativas ao seu desempenho psicossocial, autonomia, tipologia de serviços necessários e custos (Knapp \& Beecham, 1990; Mccrone et al., 1998). No nível internacional, observou-se uma relativa alteração nesse quadro na última década, com a realização de diversos estudos com escalas de avaliação em pacientes agudos e crônicos.

Um dos maiores desafios colocados para os diversos processos de reforma psiquiátrica é a transferência da assistência a pacientes graves, internados há longos anos em hospitais psiquiátricos, para alternativas de atendimento na comunidade. São pacientes afastados há tempos da sociedade e da família, submetidos aos efeitos deletérios da institucionalização prolongada, com vínculos sociais esmaecidos e portanto com dificuldade de viver sozinhos e gerenciar sua vida (Hafner, 1987; Lamb \& Peele, 1984; Mechanic, 1991).

Para esses pacientes é fundamental um processo de reabilitação psicossocial que inclua um planejamento terapêutico individua- lizado, de acordo com o nível de dependência e dificuldade no convívio social, priorizando questões como lazer, trabalho, renda e relações afetivas, que são aspectos essenciais para uma qualidade de vida satisfatória. A utilização de instrumentos padronizados permite uma avaliação objetiva desses parâmetros, que são fundamentais na vida cotidiana dos pacientes. Isso contribuirá não só na definição das intervenções adequadas e necessárias no momento, mas também na avaliação da eficácia dessas intervenções através da comparação com novas medidas em um segundo momento.

O diagnóstico psiquiátrico é habitualmente considerado um instrumento insuficiente para prever os resultados da reabilitação. Não é um bom mensurador de dependência, incapacidade ou necessidade de serviços, em função de sua baixa confiabilidade e pouca influência na determinação, reabilitação ou prognóstico da doença mental crônica (Wing, 1989; Wykes \& Sturt, 1987).

A questão da confiabilidade dos diagnósticos em psiquiatria tem sido objeto de vários estudos. Em função do pouco conhecimento sobre as origens das doenças mentais, a classificação diagnóstica não é feita a partir da etiologia, mas sim a partir de síndromes, sinais e sintomas. São classificações subjetivas e portanto pouco exatas, pois não há qualquer exame laboratorial ou teste psicológico que de fato contribua eficazmente para o diagnóstico. Podem sofrer a influência de vários fatores que interferem na sua confiabilidade, entre eles: a linha conceitual do psiquiatra, a classe social do paciente, a cultura em que estão inseridos.

Com a saída dos pacientes para a comunidade, surge a necessidade de se utilizar instrumentos para avaliar seu desempenho social, não só para ter uma medida mais objetiva de sua capacidade de sobrevivência fora do hospital como para estruturar programas de reabilitação psicossocial (Wiersma, 1996). A incapacidade na doença mental (inabilidade social) está relacionada principalmente a comportamentos e papéis desempenhados pelos pacientes em seu funcionamento social (Wykes et al., 1985).

Entre as escalas utilizadas para medir as dificuldades no comportamento social, destacam-se:

- Rehabilitation Evaluation (REHAB) - É formada por duas subescalas (adaptação social e conduta problema). Mede o nível de autono- 
mia básico do paciente e sua capacidade para adaptar-se socialmente (Baker \& Hall, 1983).

- Independent Living Skills Survey (ILSS) Proporciona uma avaliação detalhada das habilidades da vida comunitária dos pacientes (Wallace, 1986).

- Life Skills Profile (LKS) - Mede deficiências e funcionamento social. Especificamente desenhada para pacientes esquizofrênicos, de fácil manuseio, embora com poucos estudos publicados (Phelan et al., 1994).

- WHO Disablement Scale (WHO DS) - Desenvolvida para avaliação clínica e deficiências causadas por distúrbios físicos e mentais (Janca \& Sartorius, 1996).

- Social Behavioural Schedule (SBS) - Avalia as limitações do comportamento social e os resultados da reabilitação (Wing, 1989).

Tomando-se em consideração a avaliação de uma maior aplicabilidade à realidade brasileira, a sua boa adaptação a contextos de institucionalização prolongada e sua ampla utilização em outros centros de pesquisa, optouse pela utilização da SBS no presente estudo. Trata-se de um instrumento estruturado que foi desenvolvido para medir as limitações do comportamento social de pacientes de longa permanência tanto em enfermarias como em residências na comunidade. É composto de 21 itens pontuados, de zero a quatro, sendo as informações obtidas a partir das observações de um profissional que conheça bem o comportamento do paciente durante todo o mês anterior à pesquisa. Foi especificamente desenhado para refletir as dificuldades no comportamento social (Wing, 1961).

Foi traduzida para o português por Caetano (1997) e o estudo de confiabilidade da escala foi realizado em 1999, na população do Núcleo Franco da Rocha/IMASJM no Rio de Janeiro por Lima et al. Foram analisadas a confiabilidade interentrevistadores (dois entrevistadores obtêm informações de um mesmo informante) e confiabilidade interentrevistados (um entrevistador obtém informações de dois informantes sobre o mesmo paciente). Foram calculados os índices Kappa (Cohen, 1968) dos interentrevistados e interentrevistadores para todos os 21 itens da SBS. A confiabilidade da escala foi considerada alta para os dois parâmetros considerados nesse estudo.

No caso dos interentrevistados, a análise da confiabilidade na SBS mostrou alto grau de concordância e variou de $74 \%$ a $100 \%$ (com uma média de 93\%). Os índices Kappa tiveram alta significância $(\mathrm{p}<0,05)$. Apenas quatro itens apresentaram problemas: pânico e fobias, idéias bizarras, maneirismos e depressão. Apesar do índice Kappa não ser significativo nesses itens, o percentual de concordância foi alto. Em três quesitos (suicídio, comportamento destrutivo, comportamento sexual inadequado) não houve variabilidade suficiente para que o índice Kappa fosse calculado.

Em relação aos interentrevistadores, a análise de confiabilidade na SBS mostrou alto grau de concordância e variou de $74 \%$ a $100 \%$ (com uma média de 93\%). Os índices Kappa tiveram alta significância $(p<0,05)$. Apenas dois itens apresentaram problemas: incoerência do discurso e lentidão e apesar do índice Kappa não ser significativo, o percentual de concordância foi alto. Em seis deles (discurso inadequado, hostilidade, idéias bizarras, hábitos inaceitáveis, comportamento destrutivo e comportamento sexual inadequado) não houve variabilidade suficiente para que o índice Kappa fosse calculado.

O objetivo desse estudo foi avaliar as limitações no comportamento social de pacientes psiquiátricos com longo tempo de internação através do instrumento Social Behavioural Schedule (SBS).

\section{Local do estudo}

A unidade em que foi realizado esse estudo é a Unidade Franco da Rocha (NFR), uma das seis unidades do IMASJM. Abriga atualmente 200 pacientes do sexo feminino. A maior parte da clientela não possui vínculo familiar e apresenta participação social limitada. Uma pequena parcela participa das atividades terapêuticas e dos eventos culturais e sociais oferecidos. É composta de quatro pavilhões asilares antigos, abrigando cerca de 40 pacientes cada um, dois módulos residenciais denominados de Lares de Acolhimento que oferecem condições de moradia mais individualizada com 40 moradoras, sendo 20 em cada casa, e um Lar em fase final de construção (Gonçalves et al., 2000).

\section{Metodologia}

Trata-se de um estudo descritivo do comportamento social de pacientes psiquiátricos de longa permanência em instituição psiquiátri- 
ca hospitalar, utilizando a aplicação de um instrumento estruturado para esse fim - a Social Behavioural Schedule (Wykes \& Sturt, 1986).

A amostra foi calculada para uma freqüência esperada de $60 \%$ de problemas no comportamento social. O tamanho da amostra encontrado foi de 43 pessoas para um nível de confiança de 95\%. Esse cálculo foi realizado através do programa Epi-info.

Foi selecionada uma amostra aleatória simples de 46 pacientes a partir de uma população de 200 pacientes do NFR. Todas as pacientes têm história de anos ininterruptos de internação no IMASJM. As entrevistas foram realizadas por quatro entrevistadores: uma psiquiatra, uma psicóloga e duas estagiárias de psicologia. Foram escolhidos como entrevistados os profissionais que tinham mais contato com o paciente selecionado.

Os entrevistadores foram treinados durante um mês. A leitura da escala foi feita em separado e em conjunto, com discussão detalhada de cada item para que todas as dúvidas fossem resolvidas e se conseguisse homogeneidade de interpretação entre os entrevistadores.

Uma análise de dados dos prontuários das pacientes foi realizada para traçar o perfil sociodemográfico da amostra. As variáveis pesquisadas foram idade, tempo de internação, naturalidade, grau de instrução, profissão antes da internação, estado civil e diagnóstico.

$\mathrm{Na}$ avaliação dos 21 itens da SBS, o método de pontuação BSM (Mild and Severe Behaviour Problems Score) foi utilizado para determinar a presença ou ausência de problemas de comportamento social. De acordo com esse método, o problema é considerado presente quando a pontuação das respostas é 2, 3 ou 4 e ausente quando a pontuação é 0 ou 1.

Método de pontuação BSM:

0 ou $1=0$ (distúrbio de comportamento

leve (1) ou inexistente (0))

2,3 ou $4=1$ (distúrbio de comportamento moderado $(2)$ ou severo $(3,4))$

A soma do total dos quesitos com pontuação 1 fornece o BSM total de cada sujeito da amostra. A média da soma dos pontos de todos os sujeitos fornece o BSM global (BSM overall) da amostra.

Baseado em semelhanças estatísticas e clínicas, os 21 itens da SBS foram agrupados em cinco categorias por Wykes et al. (1982):

- Afastamento social: 1, 18, 19.
- Comportamento social embaraçoso: 2, 3, $4,9,11,16,20$.

- Depressão e ansiedade: 6, 7, 8, 15.

- Hostilidade e violência: 5, 14.

- Comportamento social inaceitável: 12, 13, 17, 21.

Os resultados obtidos foram comparados com os resultados de uma amostra de moradores em um albergue de sem-teto (homeless) (Hamid, 1991) e com uma amostra de pacientes de um hospital psiquiátrico de longa permanência, ambos situados em Londres (Wykes et al., 1985).

Os resultados foram discutidos em relação às 21 questões da escala (freqüência por item) e em relação ao número de problemas computados por paciente. Foi realizado o teste de Quiquadrado para verificar se as diferenças encontradas, entre as três amostras, foram significativas. Os programas estatísticos utilizados foram o Epi-info versão 6.04b e o SPSS versão 8.0.

\section{Resultados}

Além de estudar a população do NFR, os dados obtidos foram utilizados para efetuar uma comparação com dois estudos realizados em Londres, ambos utilizando o mesmo instrumento:

- Moradores de albergue de sem-teto (Hamid, 1991): uma amostra de 101 pessoas foi escolhida a partir de uma população residente em um abrigo de sem-teto. Foi composta exclusivamente por homens, em função de que estes apresentam mais problemas de distúrbios mentais do que as mulheres, e o objetivo do estudo era verificar se havia diferença significativa entre as limitações no comportamento social dessa população e as de uma amostra de pacientes psiquiátricos. A hipótese de que as limitações entre os albergados eram significativamente menores foi confirmada. Os distúrbios psiquiátricos estavam presentes em $25,7 \%$ da amostra, distribuídos da seguinte forma: $48,6 \%$ - depressão; $20 \%$ - distúrbios psicóticos; 11,4\% - demência; 8,6\% distúrbio de ansiedade; $2,8 \%$ - epilepsia. Os problemas com alcoolismo estavam presentes em $35,6 \%$ da amostra, e destes, $21 \%$ foram codificados como consumidores severos de álcool. - Pacientes de um hospital psiquiátrico de longa permanência (Wykes et al., 1985): uma amostra de 66 pacientes ( 38 homens e $28 \mathrm{mu}$ lheres) foi selecionada a partir de uma popu- 
lação de uma rede de reabilitação do Nethern Hospital e distribuída igualmente entre os diversos tipos de serviço: enfermarias, moradias protegidas e moradias na comunidade. A maioria tinha uma longa história de atendimento psiquiátrico e uma idade média de 44,5 anos. Os distúrbios psiquiátricos se distribuíram da seguinte forma: esquizofrenia - 52\%; distúrbio da personalidade - $14 \%$; depressão $12 \%$; distúrbios afetivos $-6 \%$; outros $-15 \%$.

Os dados sociodemográficos da amostra do NFR revelaram uma população com uma idade média de 64,8 anos ( $\mathrm{sd}=11,8)$ e um tempo médio de internação de 36,5 anos $(s d=8,1)$. A maioria é analfabeta $(53,5 \%)$, solteira $(69,6 \%)$, natural do Estado do Rio de Janeiro (45\%) e exercia atividades domésticas antes da internação $(80,4 \%)$. A maior prevalência é do diagnóstico de esquizofrenia (74\%), seguido dos diagnósticos de oligofrenia (13\%), epilepsia (8\%) e distúrbio bipolar (5\%).

Não houve recusas em participar das entrevistas, que duraram de 15 a 20 minutos cada uma. As questões foram bem entendidas pelos entrevistados. Embora os resultados tenham sido compatíveis com as características de uma população de pacientes crônicos (Harvey, 1996), a média dos itens da SBS pontuados com dois ou mais (BSM) foi $2.5(s d=2.9)$, um escore relativamente baixo para uma população internada em média há 36 anos sem interrupção. Nenhuma limitação foi apresentada por mais de $35 \%$ dos pacientes.

A população do NFR apresentou maiores dificuldades na área da iniciativa $(30.4 \%)$ e da aparência pessoal e higiene (30.4\%). A seguir, os problemas mais encontrados foram dificuldade de concentração $(28.3 \%)$ e inatividade $(26.1 \%)$. Os percentuais de problema em relação à conversa inadequada, comportamento suicida, realização de idéias bizarras, comportamento destrutivo, depressão e comportamento sexual inadequado foram muito baixos (menos de 2.5\%) (Tabela 1). Apesar de observarmos um percentual de $74 \%$ de esquizofrenia na amostra estudada, apenas $20 \%$ apresentaram problemas graves em relação ao comportamento social, o que reitera a importância de cotejar os resultados das avaliações do comportamento social com os diagnósticos para aferir a capacidade de convívio social dos pacientes.

\section{Tabela 1}

Quando agrupamos os resultados em relação às cinco categorias de Wykes et al., $43.5 \%$ da amostra apresentaram problemas na categoria comportamento social inaceitável, 39.1\% na categoria afastamento social, $37 \%$ na categoria comportamento social embaraçoso, $19.6 \%$ na categoria depressão e ansiedade e $8.7 \%$ na categoria hostilidade e violência.

A tabela 2 foi construída com o objetivo de comparar a prevalência de limitações dos comportamentos sociais do estudo atual com aquele conduzido por Hamid (1991) em um albergue de sem-teto em Londres e um outro conduzido por Wykes (1985) em um hospital psiquiátrico de pacientes de longa permanência também situado na cidade de Londres. Conforme pode-se observar, não há diferenças significativas na maioria dos quesitos.

\section{Tabela 2}

Os resultados mostram que a população do NFR embora internada, em média, há mais de 35 anos, apresenta comportamento social mais adequado do que os pacientes hospitalizados de Londres em vários itens: excentricidade no diálogo, inabilidade em fazer contatos sociais, hostilidade, comportamento chamativo, pânico/fobias, lentidão motora e outros comportamentos que impeçam progresso. Em relação a esses comportamentos, a população da NFR demonstra ser mais semelhante à população de sem-teto de Londres.

Há diferenças com a população dos semteto de Londres nos itens comunicação espontânea, incoerência no discurso, estereotipias, aparência pessoal e higiene, inatividade e concentração.

A população dos sem-teto tem um comportamento social mais adequado em relação a esses itens, o que é esperado porque o percentual de psicóticos neste grupo é $20 \%$ e na população do NFR é 79\% (esquizofrenia + distúrbio bipolar).

Apenas $4.3 \%$ da população do NFR apresentaram problemas quanto ao item 21 (outros comportamentos que impeçam progresso). A diferença encontrada em relação às outras duas populações se deve, provavelmente ao fato de que, nessas, a alta pontuação neste item é devida principalmente à presença de alcoolismo, distúrbio pouco freqüente entre as mulheres da unidade estudada. 
Tabela 1

Distribuição das limitações do comportamento social ${ }^{\star}$ aferidas pela SBS em uma amostra de 46 pacientes do Núcleo Franco da Rocha.

\begin{tabular}{lcc}
\hline Tipo de problema & Número & $\%$ \\
\hline 1-Comunicação: tomando a iniciativa & 14 & 30.4 \\
2-Conversação: incoerência & 7 & 15.2 \\
3-Conversação: excentricidade/inadequação & 1 & 2.2 \\
4-Interação social: habilidade de fazer contatos sociais de forma adequada & 8 & 17.4 \\
5-Interação social: proporção de contatos sociais que são por natureza hostis & 3 & 6.5 \\
6-Interaço social: comportamento chamativo & 4 & 8.7 \\
7-Idéias ou comportamento suicida ou de auto-agressão & 1 & 2.2 \\
8-Ataques de pânico e fobias & 2 & 4.3 \\
9-Inquietação e hiperatividade & 7 & 15.2 \\
10-Rir e falar sozinho & 7 & 15.2 \\
11-Realização de idéias bizarras & 1 & 2.2 \\
12-Estereotipias e maneirismos & 6 & 13 \\
13-Hábitos ou maneiras socialmente inaceitáveis & 6 & 13 \\
14-Comportamento destrutivo & 1 & 2.2 \\
15-Depressão & 1 & 2.2 \\
16-Comportamento sexual inadequado & 0 & 0 \\
17-Aparência pessoal e higiene & 14 & 30.4 \\
18-Lentidão & 2 & 4.3 \\
19-Inatividade & 12 & 26.1 \\
20-Concentração & 13 & 28.3 \\
21-Comportamento não-especificado em outro lugar que impeça progressos & 2 & 4.3 \\
\hline * Pontuaça 2ou mas
\end{tabular}

* Pontuação 2 ou mais nos 21 itens da SBS.

A tabela 3 foi construída com o objetivo de comparar o número de problemas encontrados no estudo atual com aqueles conduzidos por Hamid e Wykes. Conforme se observa, a diferença significativa se deu com o estudo de Wykes, que apresentou um percentual maior de indivíduos com 3-5 problemas, enquanto que a amostra do NFR apresentou uma maior freqüência de indivíduos sem problemas.

\section{Tabela 3}

Os resultados revelam uma população com razoável nível de convivência social. Um percentual significativo da amostra não apresentou problemas (36.9\%). A população do NFR não apresentou diferença significativa em relação ao número de problemas quando comparada à população de sem-teto de Londres. Já em relação à população de pacientes crônicos, observamos que a população brasileira apresenta uma proporção significativamente maior de indivíduos sem dificuldades e um percentual significativamente menor de indivíduos com o escore de três a cinco problemas (tabela 3 ).
Houve uma diferença estatisticamente significativa entre a amostra do NFR e a amostra de sem-teto em sete dos 21 itens da SBS e entre a primeira e a amostra de pacientes crônicos de Londres em também sete dos 21 itens da SBS.

A pontuação $B S M$ overall da amostra do presente estudo foi de 2.5 ( $\mathrm{sd}=2.9$ ). Essa média foi comparada com a dos sem-teto, que foi de 1.8 ( $\mathrm{sd}=0.14)$, e com a de população de pacientes crônicos de Londres que foi de 4.3 ( $\mathrm{sd}=0.09$ ). A diferença entre as três amostras foi estatisticamente significativa: teste $\mathrm{f}=75.4(\mathrm{p}<0.001)$.

Portanto, a população do NFR se diferencia das outras duas, e embora tenha mais problemas no desempenho social que a população dos sem-teto apresenta um comportamento social mais adequado do que a população de pacientes crônicos de Londres.

\section{Conclusão}

Apesar da faixa etária e dos longos anos de internação, um terço da população estudada apresentou características de comportamento social compatível com a convivência em ser- 
Tabela 2

Comparação percentual entre os 21 itens da SBS no estudo atual e nos estudos realizados por Hamid e Wykes.

\begin{tabular}{|c|c|c|c|}
\hline Tipo de problema & $\begin{array}{c}\text { NFR \% } \\
\mathrm{n}=46\end{array}$ & $\begin{array}{c}\text { Hamid \% } \\
\mathrm{n}=101\end{array}$ & $\begin{array}{l}\text { Wykes \% } \\
\mathrm{n}=66\end{array}$ \\
\hline 1-Comunicação: tomando a iniciativa & 30,4 & $5(\chi: 18,24 ; p=0,00001)$ & $26 \mathrm{NS}^{*}$ \\
\hline 2-Conversação: incoerência & 15,2 & $2^{\star}(\chi: 7,44 ; p=0,0006)$ & $14 \mathrm{NS}^{*}$ \\
\hline 3-Conversação:excentricidade/inadequação & 2,2 & $6,9 \mathrm{NS}^{*}$ & $26(\chi: 6,02 ; p=0,0006)$ \\
\hline $\begin{array}{l}\text { 4-Interação social: habilidade de fazer contatos } \\
\text { sociais de forma adequada }\end{array}$ & 17,4 & $14,9 \mathrm{NS}^{*}$ & $38(\chi: 6,02 ; \mathrm{p}=0,01)$ \\
\hline $\begin{array}{l}\text { 5-Interação social: proporção de contatos } \\
\text { sociais que são por natureza hostis }\end{array}$ & 6,5 & $17,8 \mathrm{NS}^{*}$ & $21(\chi: 4,71 ; p=0,02)$ \\
\hline 6-Interação social: comportamento chamativo & 8,7 & $8,9 \mathrm{NS}^{*}$ & $27(\chi: 6,18 ; p=0,01)$ \\
\hline 7-Idéias ou comportamento suicida ou de auto-agressão & 2,2 & $2 \mathrm{NS}^{*}$ & $9 \mathrm{NS}^{*}$ \\
\hline 8-Ataques de pânico e fobias & 4,3 & $6,9 \mathrm{NS}^{*}$ & $30(\chi: 11,93 ; p=0,0005)$ \\
\hline 9-Inquietação e hiperatividade & 15,2 & $9,9 \mathrm{NS}^{*}$ & $14 \mathrm{NS}^{*}$ \\
\hline 10-Rir e falar sozinho & 15,2 & $7,9 \mathrm{NS}^{*}$ & $23 \mathrm{NS}^{*}$ \\
\hline 11-Realização de idéias bizarras & 2,2 & $4 \mathrm{NS}^{*}$ & $6 \mathrm{NS}^{*}$ \\
\hline 12-Estereotipias e maneirismos & 13,0 & $3(\chi: 3,06 ; p=0,04)$ & $15 \mathrm{NS}^{*}$ \\
\hline 13-Hábitos ou maneiras socialmente inaceitáveis & 13,0 & $5 \mathrm{NS}^{*}$ & $15 \mathrm{NS}^{*}$ \\
\hline 14-Comportamento destrutivo & 2,2 & $5 \mathrm{NS}^{*}$ & $6 \mathrm{NS}^{*}$ \\
\hline 15-Depressão & 8,7 & $9,9 \mathrm{NS}^{*}$ & $12 \mathrm{NS}^{*}$ \\
\hline 16-Comportamento sexual inadequado & 0 & $2 \mathrm{NS}^{*}$ & $2 \mathrm{NS}^{\star}$ \\
\hline 17-Aparência pessoal e higiene & 30,4 & $11,9(\chi: 7,47 ; p=0,006)$ & $39 \mathrm{NS}^{*}$ \\
\hline 18-Lentidão & 4,3 & $5 \mathrm{NS}^{*}$ & $23(\chi: 7,60 ; \mathrm{p}=0,005)$ \\
\hline 19-Inatividade & 26,1 & $13,9(\chi: 3,24 ; \mathrm{p}=0,07)$ & $17 \mathrm{NS}^{*}$ \\
\hline 20-Concentração & 28,3 & $5(\chi: 15,98 ; p=0,00006)$ & $17 \mathrm{NS}^{*}$ \\
\hline $\begin{array}{l}\text { 21-Comportamento não-especificado } \\
\text { em outro lugar que impeça progressos }\end{array}$ & 4,3 & $35,6(\chi: 16,15 ; \mathrm{p}=0,0005)$ & $36(\chi: 16,5 ; p=0,0005)$ \\
\hline
\end{tabular}

\footnotetext{
${ }^{*} \mathrm{NS}=\mathrm{p}>0,05$
}

Tabela 3

Comparação das freqüências (\%) dos problemas de comportamento social na SBS entre o estudo atual e nos realizados por Hamid e Wykes

\begin{tabular}{lccc}
\hline Problemas SBS & NFR $(\mathbf{n}=\mathbf{4 6})$ & Hamid $(\mathbf{n}=\mathbf{1 0 1})$ & Wykes $(\mathbf{n}=\mathbf{6 6})$ \\
\hline Sem problemas & 36,9 & $42,6(\chi: 0,41 ; \mathrm{p}=0,52)$ & $9,1(\chi: 12,40 ; \mathrm{p}=0,003)$ \\
$1-2$ problemas & 28,3 & $29,7(\chi: 0,03 ; \mathrm{p}=0,85)$ & $21,2(\chi: 0,74 ; \mathrm{p}=0,39)$ \\
$3-5$ problemas & 15,2 & $17,8(\chi: 0,15 ; \mathrm{p}=0,69)$ & $39,4(\chi: 7,62 ; \mathrm{p}=0,005)$ \\
$>5$ problemas & 19,6 & $9,9(\chi: 2,62 ; \mathrm{p}=0,10)$ & $30,3(\chi: 1,63 ; \mathrm{p}=0,20)$ \\
\hline
\end{tabular}

$\mathrm{NS}=\mathrm{p}>0,05$

viços residenciais terapêuticos, reforçando a necessidade de transferência dos cuidados a esses pacientes do hospital para a comunidade. Além disso, $65 \%$ da população se mostrou apta a residir em moradias na comunidade, pois apresentou apenas 1 a 2 problemas na escala SBS.

Os pacientes mais graves perfazem um total de 19,6\%, o que indica que essa clientela necessita de projetos terapêuticos específicos, que também levem em conta questões relativas à qualidade de vida e à autonomia.

Encontramos na amostra do NFR um percentual significativamente menor de pacientes com dificuldades sociais do que a população de pacientes de longa permanência de Londres. Tal discrepância talvez possa ser explicada pelo fato da Inglaterra ter iniciado seu processo de desinstitucionalização há mais de quatro décadas e, portanto, os pacientes que 
permanecem institucionalizados podem ser os mais refratários às estratégias de reabilitação psicossocial.

A comparação da amostra com as duas populações de Londres deve ser feita com reservas, em função das diferenças culturais entre os dois países, das diferenças em relação à prevalência de distúrbios mentais, ao gênero, à presença de alcoolismo nas duas populações de Londres, e em função da Inglaterra estar bem à frente do Brasil em seu processo de desinstitucionalização psiquiátrica. Apesar disso, essas comparações se tornam fundamentais pela escassez de estudos na área. Naturalmente, se tivéssemos no Brasil outros estudos com pacientes internados em hospitais psiquiátricos, avaliados pelo mesmo instrumento, estes seriam o grupo de comparação ideal.

É importante que sejam iniciadas pesquisas em relação à necessidade e qualidade dos serviços a serem oferecidos aos pacientes que possam ser transferidos para o convívio comunitário e planejamento individualizado para tratamento e reabilitação psicossocial. Entre os problemas a serem enfrentados na tentativa de reinserir pacientes graves na vida social, a questão da autonomia aparece como um fator primordial em situações como: gerenciar dinheiro, cuidados com a aparência e a casa, fazer compras.

As instituições hospitalares brasileiras, em sua maioria, não oferecem aos pacientes programas específicos dirigidos à reabilitação psicossocial, assim como não dispõem de condições adequadas que garantam o respeito à individualidade e o direito ao exercício de um convívio social digno.

O grande desafio está na construção de uma rede de alternativas à internação psiquiátrica que estejam de acordo com as necessidades dos pacientes que estão sendo desinstitucionalizados. É fundamental que haja investimento político e aplicação de recursos na criação de serviços residenciais terapêuticos com níveis de proteção diferenciados de acordo com a autonomia de cada grupo.

As novas modalidades de atenção, uma vez implantadas, devem ser periodicamente avaliadas nos seus processos e resultados, mantendo um compromisso ético com a assistência que atenda aos interesses dos indivíduos como sujeitos sociais e garantam seu direito de cidadania.

\section{Referências bibliográficas}

Baker R, Hall JN 1983. Rehabilitation evaluation of Hall and Baker (REHAB). Vine Publishing, Aberdeen.

Bandeira M 1991. Desinstitucionalização ou transinstitucionalização: lições de alguns países. Jornal Brasileiro de Psiquiatria 40(7): 355-360.

Bassuk EL, Gerson S 1 978. Deinstitutionalization and mental health services. Scient Am 238: 46-53.

Braun P, Kochansky G, Shapiro R, Greenberg S, Gudeman JE, Johnson S \& Shore MF 1981. Overview: deinstitutionalization of psychiatric patients: a critical review of outcome studies. Am J Psychiatry 138 (6): 736-749.

Caetano GV 1997. Bloqueio de leitos e suas conseqüências na prestação de serviços na área. Dissertação de mestrado. Escola Nacional de Saúde Pública, Fundação Oswaldo Cruz, Rio de Janeiro. 
Cohen J 1968. Weighted Kappa: nominal scale agreement with provisions for scales disagreement of partial credit. Psychological Bulletin 70: 213-20.

Fagundes P 1998. Desinstitucionalização da assistência psiquiátrica: algumas questões cruciais. Jornal Brasileiro de Psiquiatria (4): 163-168.

Gonçalves SR, Assumpção AM, Barbosa DR, Santos MM, Freitas RC, Nigri F, Rufino C, Pinto MMB, Domeni R, Keusen A \& Lima LA 2000. Lar de acolhimento: implantação de um módulo residencial. Enviado para publicação no Jornal Brasileiro de Psiquiatria.

Hafner A 1987 . Do we still need beds for psychiatric patients? Acta Psychiatrica Scandinavia 75:113-126.

Hafner H, Heiden W 1989. The evaluation of mental health care system. Br J Psych 155: 12-17.

Hamid WKA 1991. Homeless people and community care: an assessment of the needs of homeless people for mental health services. Thesis of $\mathrm{PhD}$. University of London.

Harvey CA 1996. The Camden schizophrenia surveys I. The psychiatric, behavioural and social characteristics of the severely mentally ill in an inner London Health District. Br J Psych 168: 410-417.

Janca A, Sartorius N 1996. Psychiatric assessment instruments developed by the World Health Organization. Soc Psychiatry Psychiatr Epidemiol 31: 5-69.

Knapp M, Beecham J 1990. Costing mental health services. Psych Med 20: 893-908.

Lamb HR, Peele R 1984. The need for continuing asylum and sanctuary. Hosp Com Psychiatr 35: 798-801.

Mccrone P, Thornicroft G, Phelan M, Holloway F, Wykes T \& Johnson S 1998. Utilization and costs of community mental health services. Br J Psych 173: 391398
Mechanic D 1991. Recent development in mental heath: perspectives and services. Annu. Rev. Publ. Health 12: 1-15.

Morgado AF, Lima LA 1994. Desinstitucionalização, suas bases e a experiência internacional. Jornal Brasileiro de Psiquiatria 43(1): 19-28.

Phelan M, Wykes T, Goldman H 1994. Global function scales. Social Psychiatry and Psychiatric Epidemiology 29: 205-211.

Wallace CJ 1986. Functional assessment in rehabilitation. Schiz Bull 12: 604-630.

Wiersma D 1996. Measuring social disabilities in mental health. Soc Psychiatry Psychiatr Epidemiol 31: 101-108

Wing JK 1961. A simple and reliable subclassification of chronic schizophrenia. Journal of Mental Science 107: 826-875.

Wing JK 1989. The measurement of "social disablement": the MRC social behaviour and social role performance schedules. Soc Psychiatry Psychiatr Epidemiol 24:173-178.

Wykes T, Sturt E \& Creer C 1982. Practices of day and residential units in relation to the social behaviour of attenders. Psychological Medicine Monograph Supplement 2. Edited by Wing JK. Cambridge University Press, Cambridge.

Wykes T, Sturt E \& Creer C 1985. The assessment of patients needs for community care. Social Psychiatry 20(2): 76-85.

Wykes T, Sturt E 1986. The measurement of social behaviour in psychiatric patients: An assessment of the reliability and validity of the SBS schedule. $\mathrm{Br} J$ Psych 148: 1-11.

Wykes T, Sturt E 1987. Assessment schedule for chronic psychiatric patients. Psychological Medicine 17: 485-493. 